\title{
Leptohyphidae (Insecta, Ephemeroptera) do Estado do Amazonas, Brasil: novos registros, nova combinação, nova espécie e chave de identificação para estágios ninfais
}

\author{
Enide Luciana Lima Belmont ${ }^{1}$, Frederico Falcão Salles ${ }^{2} \&$ Neusa Hamada ${ }^{1,3}$
}

\begin{abstract}
'Programa de Pós-Graduação em Entomologia, Instituto Nacional de Pesquisas da Amazônia, Avenida André Araújo, 2936, 69067-375 Manaus-AM, Brasil.belmont@inpa.gov.br

${ }^{2}$ Universidade Federal do Espírito Santo, BR 101 km 60, Bairro Litorâneo, 29932-540 São Mateus-ES, Brasil. ffsalles@gmail.com

${ }^{3}$ Coordenação de Biodiversidade, Instituto Nacional de Pesquisas da Amazônia, Avenida André Araújo, 2936, 69067-375 Manaus-AM, Brasil. nhamada@inpa.gov.br
\end{abstract}

\begin{abstract}
Leptohyphidae (Insecta, Ephemeroptera) of Amazonas state, Brazil: new records, new combination, new species and identification key for nymphal stages. The following genera of Leptohyphidae occur in the Amazonas state: Amanahyphes Salles \& Molineri, Leptohyphes Eaton, Tricorythodes Ulmer and Tricorythopsis Traver. Distribution of Leptohyphidae species in Amazonas state is presented. A new species, Tricorythodes yapekuna sp. nov., is described and can be distinguished from other Tricorythodes by: (1) tarsal claws with pair of submarginal denticles and no marginal denticles; (2) bi-articulated maxillary palp; (3) opercular gill black except on apical margin; (4) gill formula 2/3/3/3/2; and (5) expanded lateral abdominal margin of segments III-VI. The new combination, Tricorythopsis rondoniensis (Dias, Cruz \& Ferreira, 2009) comb. nov., is proposed and constitutes the first record of this species in the Amazonas State. An illustrated dichotomous key to identify nymphs of genera and species occurring in the Amazonas State is also presented.
\end{abstract}

KEYWORDS.Aquatic insects; Ephemerelloidea; Presidente Figueiredo; Reserva Florestal Ducke.

RESUMO. Leptohyphidae (Insecta, Ephemeroptera) do Estado do Amazonas, Brasil: novos registros, nova combinação, nova espécie e chave de identificação para estágios ninfais. Os seguintes gêneros de Leptohyphidae ocorrem no estado do Amazonas: Amanahyphes Salles \& Molineri, Leptohyphes Eaton, Tricorythodes Ulmer e Tricorythopsis Traver. A distribuição das espécies de Leptohyphidae no Estado do Amazonas é apresentada. Uma espécie nova, Tricorythodes yapekuna sp. nov., é descrita e pode ser diferenciada de outros Tricorythodes pelas (1) garras tarsais com um par de dentículos submarginais e sem dentículos marginais; (2) palpo maxilar biarticulado; (3) brânquia opercular uniformemente preta com exceção da margem apical; (4) fórmula branquial 2/3/3/3/2; e (5) margem lateral do abdome expandida nos segmentos III-VI. Uma combinação nova, Tricorythopsis rondoniensis (Dias, Cruz \& Ferreira, 2009) comb. nov., é proposta e constitui o primeiro registro dessa espécie para o Estado do Amazonas. Uma chave dicotômica ilustrada para identificar ninfas de gêneros e espécies ocorrentes no Amazonas também é apresentada.

PALAVRAS-CHAVE. Ephemerelloidea; Insetos aquáticos; Presidente Figueiredo; Reserva Florestal Ducke.

Leptohyphidae tem distribuição Pan-americana com cerca de 140 espécies distribuídas em 10 gêneros (Molineri 2010). No Brasil, é representada por sete gêneros e 41 espécies (Salles et al. 2011), sete destas têm registro para o Estado do Amazonas: Amanahyphes saguassu Salles \& Molineri, 2006, Leptohyphespopulus Allen, 1973, Tricorythopsis acara Belmont, Salles \&Hamada, 2011, Tricorythopsis bahiensis Dias, Salles \& Ferreira, 2008, Tricorythopsis intercalatus Belmont, Salles \&Hamada, 2011, Tricorythopsis yucupe Dias, Salles \& Ferreira, 2008 e Tricorythodes faeculopsis Belmont, Salles \& Hamada, 2011. Poucas informações estão disponíveis sobre a ecologia e a biologia desta família (Edmunds et al. 1976; Molineri 2001). As ninfas são normalmente robustas, têm corpo coberto por cerdas, brânquias operculares no segmento II que cobrem as restantes e filamentos caudais tão longos quanto o corpo. Vivem em habitats lóticos, utilizando diversos substratos nos cursos d'água, mas preferencialmente são encontradas em areia e cascalho (Molineri 2010). Quanto à categoria funcional trófica, as ninfas são classificadas como "coletoras de depósito" de sedimento (Cummins et al. 2005; Molineri 2010). As subimagos emergem durante a noite e transformam-se em adultos antes do amanhecer (Molineri 2010). Os adultos são pequenos, têm tórax robusto, asas anteriores com cerdas na margem posterior, asas posteriores, quando presentes, são pequenas e possuem uma longa projeção costal; o pênis varia na forma; fórceps, com 2 ou 3 artículos (Domínguez et al. 2006).

Este estudo foi desenvolvido com o objetivo de ampliar o conhecimento sobre as espécies de Leptohyphidae conhecidas para o Estado do Amazonas, fornecer informações sobre a bionomia e distribuição delas, além de elaborar uma chave dicotômica ilustrada para identificar estágios ninfais de gêneros e espécies. Apesar do caráter preliminar, a chave proposta será de grande relevância, pois integra os caracteres diagnósticos dos táxons registrados até o momento e servirá de ferramenta para pesquisadores que estão iniciando trabalhos com insetos aquáticos na região. Contudo, pelo fato da abrangência geográfica desse estudo no Estado do Amazonas ter sido pe- 
quena em virtude da grande extensão territorial, ressaltamos que espécies, ou mesmo gêneros previamente descritos, ainda devem ser encontrados no estado, além, é claro, de táxons desconhecidos para a Ciência. Dessa forma, a chave deve ser utilizada com cautela e as identificações devem ser confirmadas acessando as revisões dos gêneros ou descrições originais.

\section{MATERIAL E MÉTODOS}

Informações sobre a distribuição das espécies foram obtidas por meio da literatura disponível (e.g., Molineri 2001; Domínguez et al. 2006; Molineri, 2006; Dias et al. 2007; Salles et al. 2011) e coletas realizadas nos meses de outubro de 2009 e março, abril e setembro de 2010. Os cursos d'água amostrados estão localizados nos municípios de Manaus e Presidente Figueiredo, Amazonas, Brasil.

No município de Manaus, as coletas foram realizadas em igarapés localizados na Reserva Florestal Ducke (AM-010, km 26) e em um balneário localizado na BR-174, km 18 (Tabela I). No município de Presidente Figueiredo as coletas foram realizadas em igarapés próximos às principais estradas de acesso, as rodovias BR-174 e AM-240 (Tabela II).

Tabela I. Locais de coletas no município de Manaus, Amazonas, Brasil. RFD = Reserva Florestal Ducke (RFD); Localização nas trilhas da RFD $\mathrm{L}-\mathrm{O}=$ Leste-Oeste; $\mathrm{N}-\mathrm{S}=$ Norte-Sul; número $=$ número da trilha; $\mathrm{m}=$ distância entre os pontos (PPBio 2011); (0256’21'”N, 59॰57’43”W).

\begin{tabular}{lll}
\hline Ponto & \multicolumn{1}{c}{ Local } & \multicolumn{1}{c}{ Localização } \\
\hline RFD1 & Igarapé Tinga 33 & L-O 3: $8.400 \mathrm{~m}$ \\
RFD2 & Igarapé Tinga 31 & N-S 7: $4.500 \mathrm{~m}$ \\
RFD3 & Igarapé Tinga 22 & N-S 7: $2.975 \mathrm{~m}$ \\
RFD4 & Igarapé Tinga 14 & N-S 6: $4.500 \mathrm{~m}$ \\
RFD5 & Igarapé Acará 32 & L-O 3: $2.900 \mathrm{~m}$ \\
RFD6 & Igarapé Acará 23 & L-O 4: $1.950 \mathrm{~m}$ \\
RFD7 & Igarapé Acará 14 & L-O 3: $1.950 \mathrm{~m}$ \\
RFD8 & Igarapé Barro Branco 21 & N-S 1: $0.100 \mathrm{~m}$ \\
RFD9 & Igarapé Ipiranga 22 & N-S 7: 7.800 m \\
RFD10 & Igarapé Ipiranga 12 & L-O 7: $6.100 \mathrm{~m}$ \\
BR 1 & Igarapé sem nome & BR 174/km 18,5 \\
\hline
\end{tabular}

As coletas foram feitas com o auxílio de uma rede em D para as ninfas e para os adultos foi usada uma armadilha luminosa do tipo Pensilvânia. Ninfas com tecas alares bem desenvolvidas e enegrecidas foram mantidas vivas, acondicionadas em recipientes com água, até a emergência do adulto, para possibilitar a associação entre estes estágios.

Nos resultados, PF refere-se a pontos de coletas no município de Presidente Figueiredo; RFD aos pontos de coletas em Manaus, na Reserva Florestal Ducke e BR a um ponto ao longo da BR-174. Entre parênteses apresentamos o número de indivíduos seguido do estágio examinado, ninfas $(\mathrm{N})$ e adultos $(\mathrm{A})$.

A chave para identificação de gêneros e espécies ocorrentes no Amazonas foi confeccionada no modelo dicotômica ilustrada. A qual é direcionada para as ninfas, pois das nove espécies registradas para essa região apenas duas tem as formas adultas descritas. Imagens das estruturas utilizadas na chave foram elaboradas com o auxílio de uma câmara clara acoplada a um estereomicroscópio e microscópio óptico, e posteriormente vetorizadas com o programa Adobe ${ }^{\circledR}$ Illustrator $^{\circledR}$. Foi elaborado um mapa com a distribuição conhecida das espécies pelo Estado do Amazonas (Fig. 1). Os espécimes examinados estão depositados na Coleção de Invertebrados do Instituto Nacional de Pesquisas da Amazônia, Amazonas, Brasil e na Coleção de Zoologia do Programa de Pós-graduação em Biodiversidade Tropical, Espírito Santo, Brasil.

\section{RESULTADOS}

\section{Amanahyphes saguassu Salles \& Molineri, 2006}

Estágios descritos: ninfa, subimago e imago macho.

Biologia: A ninfas são encontradas em raízes, areia, vegetação marginal, junto com diversas outras ninfas de Ephemeroptera. Também podem ser encontradas em igarapés com laje e pedras.

Distribuição: Brasil: Amazonas (Manaus, Presidente Figueiredo) (Salles \& Molineri 2006); Pará (Parauapebas) (Gonçalves e Da-Silva 2010); Venezuela: Bolivar (Tarota) (Molineri et al. 2011).

Tabela II. Locais de coletas no município de Presidente Figueiredo, Amazonas, Brasil e coordenadas geográficas. (R.Ec. = Reserva Ecológica).

\begin{tabular}{|c|c|c|c|c|}
\hline Ponto & Local & Localização & \multicolumn{2}{|c|}{ Coordenadas } \\
\hline PF1 & Ramal Paulo Freire: Sítio os Pioneiros & AM 240/km 5 & $02^{\circ} 04^{\prime} 5,0^{\prime \prime} \mathrm{S}$ & $59^{\circ} 54^{\prime} 28,8^{\prime \prime} \mathrm{O}$ \\
\hline PF2 & Caverna Refúgio do Maroaga & AM 240/km 11 & $02^{\circ} 02^{\prime} 57^{\prime \prime} \mathrm{S}$ & $59^{\circ} 58^{\prime} 29 ” O$ \\
\hline PF3 & R.Ec. Cachoeira Santuário & AM 240/km 12 & $02^{\circ} 03^{\prime} 43^{\prime \prime S}$ & $59^{\circ} 55^{\prime} 44^{\prime \prime} \mathrm{O}$ \\
\hline PF 4 & Pousada Rio Belo & AM $240 / \mathrm{km} 12,5$ & $02^{\circ} 03^{\prime} 13^{\prime \prime} \mathrm{S}$ & $59^{\circ} 55^{\prime} 25,8^{\prime} \mathrm{O}$ \\
\hline PF 5 & Cachoeira da Porteira & AM 240/km 13 & $02^{\circ} 02^{\prime} 15^{\prime \prime} \mathrm{S}$ & $59^{\circ} 54^{\prime} 53 ” \mathrm{O}$ \\
\hline PF 6 & Cachoeira Maroca & AM 240/km 17 & $02^{\circ} 00^{\prime} 57,1^{\prime} \mathrm{S}$ & $59^{\circ} 51^{\prime} 32,7^{\prime} \mathrm{O}$ \\
\hline PF 7 & Corredeira Santo Amaro & AM 240/km 20 & $02^{\circ} 04^{\prime} 05,0 ” \mathrm{~S}$ & $59^{\circ} 54^{\prime} 28,8^{\prime} \mathrm{O}$ \\
\hline PF 8 & Balneário Sossego da Pantera, igarapé da onça & AM 240/km 20 & $02^{\circ} 02^{\prime} 34^{\prime \prime} \mathrm{S}$ & $59^{\circ} 51^{\prime} 08^{\prime \prime} \mathrm{O}$ \\
\hline PF 9 & Sítio do Seu José, igarapé da Ponte & AM 240/km 24 & $02^{\circ} 01^{\prime} 05^{\prime} \mathrm{S}$ & $59^{\circ} 43^{\prime} 25,7^{\prime} \mathrm{O}$ \\
\hline PF10 & Cachoeira da Pedra Furada & AM 240/km 57 & $01^{\circ} 59^{\prime} 34,0 ” S$ & $59^{\circ} 33^{\prime} 26,4^{\prime \prime} \mathrm{O}$ \\
\hline PF 11 & Corredeira do Barreto & AM 240/km 65 & $01^{\circ} 58^{\prime} 04^{\prime \prime} \mathrm{S}$ & $59^{\circ} 29^{\prime} 24^{\prime \prime} \mathrm{O}$ \\
\hline PF 12 & Residencial Bosque das Águas & BR 174/km 1 & $02^{\circ} 02^{\prime} 42,8^{\prime \prime} \mathrm{S}$ & $60^{\circ} 01^{\prime} 20,3{ }^{\prime \prime} \mathrm{O}$ \\
\hline PF 13 & Hotel Fazenda Marupiara & BR 174/km 107 & $02^{\circ} 07^{\prime} \mathrm{S}$ & $60^{\circ} 06^{\prime} \mathrm{O}$ \\
\hline
\end{tabular}




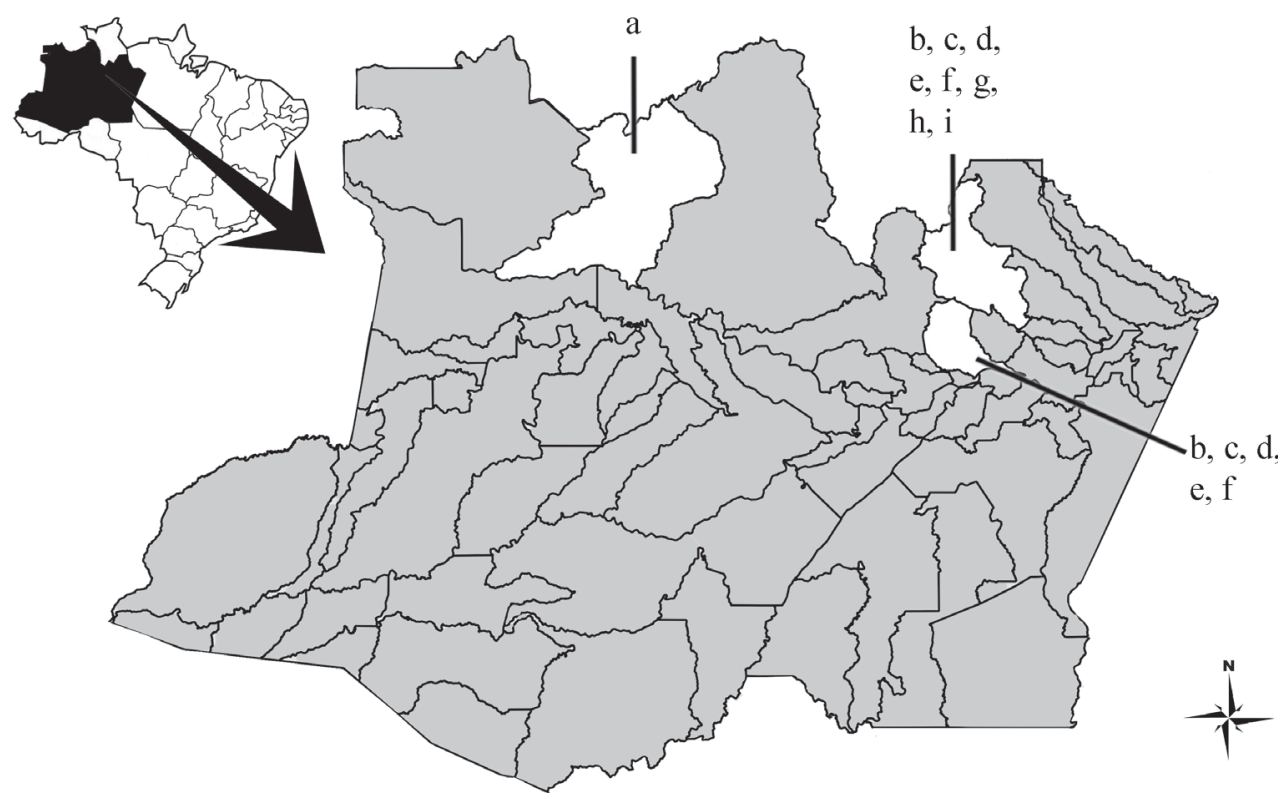

Fig. 1. Distribuição das espécies de Leptohyphidae registradas no Estado do Amazonas, Brasil. (a- Leptohyphes populus; b- Amananhyphes saguassu; cTricorythodes faeculopsis; d- Tricorythodes yapekuna sp. nov.; e- Tricorythopsis rondoniensis; f- Tricorythopsis acara; g- Tricorythopsis bahiensis; hTricorythopsis intercalatus; i- Tricorythopsis yucupe).

Material Examinado:PF1: (10N) 12.xi.2009; PF3: (8N) 13.xi.2009; PF6: (11N) 10.xi.2009; PF7: (16N) 09.xi.2009; PF8: (11N) 09.xi.2009; PF9: (6N) 16.xi.2009; PF12: (1N) 14.xi.2009; PF13: (3N) 15.xi.2009; RFD2: (22N) 27.iii.2010; RFD3: (23N) 27.iii.2010; RFD4: (14N) 28.iii.2010; RFD5: (2N) 12.iv.2010; RFD6: (16N) 12.iv.2010; RFD7: (36N) 13.iv.2010; RFD8: (12N) 13.iv.2010; RFD9: (3N) 10.v.2010.

\section{Leptohyphes populus Allen, 1973}

Estágio descrito: ninfa.

Biologia: Não há informações dessa natureza sobre a espécie.

Distribuição: Brasil: Amazonas (Santa Isabel do Rio Negro).

Material Examinado: Essa espécie não foi coletada durante o desenvolvimento deste trabalho. A distribuição é baseada na literatura (Allen 1973; Hubbard 1982; Molineri 2003; Domínguez et al. 2006).

\section{Tricorythodes faeculopsis}

Belmont, Salles \& Hamada, 2011

Estágios descritos: ninfa e subimago macho.

Biologia:As ninfas são encontradas em raízes finas com bastante depósito de sedimento. Estas são facilmente reconhecidas por ter seu corpo coberto de sedimento que fica preso às cerdas.

Distribuição: Brasil: Amazonas (Manaus, Presidente Figueiredo) (Belmont et al. 2011).

Material Examinado:PF1: (2N) 12.xi.2009; PF8: (1S) 09.xi.2009; PF10: (1N) 11.xi.2009; RFD1: (1N); RFD1: (3N) 27.iii.2010; RFD 4: (3N) 28.iii.2010; RFD5: (1N) 12.iv.2010; RFD6: (1N) 12.iv.2010; RFD8: (1N) 13.iv.2010; RFD9: (14N) 10.v.2010.
Tricorythodes yapekuna sp. nov.

(Figs. 2-13)

Estágio descrito: ninfa.

Biologia: As ninfas são encontradas em raízes e vegetação nas margens dos igarapés.

Distribuição: Brasil: Amazonas (Manaus, Presidente Figueiredo).

Material Examinado: PF3: (2N) 13.xi.2009; PF4: (3N) 01.ix.2010; PF9: (3N) 16.xi.2009; RFD9: (1N) 10.v.2010; RFD10: (9N) 16.xi.2009; BR1: (11N) 09.x.2009.

Descrição: Ninfa madura. Comprimento: corpo, 2,6-3,0 $\mathrm{mm}$; mesonoto, 0,9-1,3 mm; filamentos caudais, 1,7-1,9 mm. Aspecto geral. Corpo alongado com muitas certas; coloração marrom amarelada. Cabeça: pigmentação marrom entre os ocelos; ausência de projeção genal, frontal e tubérculos; aparelho bucal amarelado com partes escurecidas; labro com uma profunda emarginaçãoanteromediana, dorsalmente com cerdas pectinadas na porção anterior e ventralmente com cerdas simples na margem externa, porção mediana e anterior (Fig. 2); hipofaringe: com cerdas pectinadas nas línguas e na superlíngua com cerdas pectinadas e simples; lábio: glossa com cerdas pectinadas grossas e finas; paraglossae com cerdas pectinadas finas; palpos labiais com cerdas simples (Fig. 3); palpos maxilares biarticulados com cerda curta (Fig. 6). Tórax: coloração amarelada com manchas marrons; ausência de tubérculos no pronoto; porção anterolateral do pronoto não projetada; pernas amareladas sem manchas, com cerdas intermediárias; coxas sem projeções; fêmures sem manchas, estreito (duas vezes mais longo que largo); fileira de cerdas na 
porção submediana dos fêmures anteriores (Fig. 4); tíbias sem manchas, com cerdas longas na porção ventral e cerdas tipo cabelo na porção dorsal (Figs. 4-5); garras tarsais longas, sem dentículos marginais e um par de dentículos submarginais. Abdome: amarelado com os segmentos pigmentados de preto; margens laterais expandidas nos segmentos III-VI (Fig. 7); segmento VII pontudo; ausência de espinhos dorsais; brânquias operculares triangulares, uniformemente manchadas de preto com exceção da margem apical (Fig. 8); fórmula branquial 2/ 3/3/3/2 (Figs. 9-13); filamentos caudais amarelados com cerdas entre os segmentos.

Diagnose: (1) garras tarsais com um par de dentículos submarginais e sem dentículos marginais; (2) palpo maxilar biarticulado; (3) brânquia opercular uniformemente preta com exceção da margem apical; (4) fórmula branquial $2 / 3 / 3 / 3 / 2$; (5) margem lateral do abdome expandida nos segmentos III-VI.

Discussão: As ninfas de T. yapekuna sp. nov. são semelhantes às ninfas de T. ocellus Allen \& Roback, 1969 e $T$. uniandinus Emmerich, 2007, duas espécies conhecidas da região andina, respectivamente do Peru e Colômbia. Tricorythodes yapekuna sp. nov. pode ser diferenciada de T. ocellus por não possuir os ocelos laterais tão desenvolvidos quanto nesta espécie e de $T$. uniandinus pela denticulação da garra tarsal, que nessa espécie é de 8-11 dentículos marginais além de um par de pequenos dentículos submarginais.

Etimologia: yapekuna (yapeku - brânquia; un/una - preto (a)) significa brânquia preta na língua Tupi-Guarani.

Material tipo: Holótipo - ninfa (em álcool), Brasil, Amazonas, Presidente Figueiredo, AM 240, km 12, Reserva Ecológica Cachoeira do Santuário, (02 03’ 43" S/59॰ 55’ 44” O), x-2009, Belmont, E.L.L.; Cruz, P.V.; Boldrini, R. col. (INPA). Parátipos - 3 ninfas (em álcool) mesmos dados do holótipo (INPA); 2 ninfas (em álcool) mesmos dados do holótipo (CEUNES).

\section{Tricorythopsis acara Belmont, Salles \& Hamada, 2011}

Estágios descritos: ninfa e imago macho.

Biologia: Encontradas em raízes finas nas margens dos igarapés, com o hábito de rastejar.

Distribuição: Brasil: Amazonas (Manaus e Presidente Figueiredo) (Belmont et al. 2011).

Material Examinado: PF1: (3N) 12.xi.2009; PF2: (1N) 12.xi.2009; PF5: (1N) 10.xi.2009; PF8: (42N) 09.xi.2009; PF10: (1N) 11.xi.2009; PF12: (2N) 14.xi.2009; PF13: (2N) 15.xi.2009; RFD5: (1N) e (1A) 12.iv.2010; RFD6: (5N) 12.iv.2010; RFD8: (8N) 13.iv.2010; RFD9: (6N) 11.v.2010.

\section{Tricorythopsis bahiensis Dias, Salles \& Ferreira, 2008}

Estágio descrito: ninfa.

Biologia: Podem ser encontradas sob pedras (cascalho), em áreas calmas do curso d'água.

Distribuição: Brasil: Amazonas (Presidente Figueiredo); Bahia (Correntina); Roraima (Bonfim) (Dias et al. 2008).

Material Examinado: PF3: (1N) 13.xi.2009; PF8: (12N) 09.xi.2009.

\section{Tricorythopsis intercalatus \\ Belmont, Salles \& Hamada, 2011}

Estágio descrito: ninfa

Distribuição: Brasil: Amazonas (Presidente Figueiredo).

Biologia: São encontrados em igarapés com fundo de laje e em raízes marginais.

Material Examinado:PF2: (1N) 12.xi.2009; PF3: (1N) 13.xi.2009; PF4: (2N) 01.ix.2010; PF5: (7N) 10.xi.2009.

\section{Tricorythopsis rondoniensis}

(Dias, Cruz \& Ferreira, 2009) comb. nov.

Tricorythodes rondoniensis Dias, Cruz \& Ferreira, 2009: 127-129, figs. 1-13

Estágio descrito: ninfa.

Biologia: As ninfas podem ser encontradas nas raízes finas em igarapés com correnteza.

Distribuição: Brasil: Amazonas (Manaus e Presidente Figueiredo); Rondônia (Porto velho) e Roraima (Boa Vista e Amajari) (Dias et al. 2009).

Comentário: Primeiro registro dessa espécie para o Amazonas.

Diagnose: (1) fêmures largos, circular e com margens cobertas por longas cerdas; (2) garras tarsais com 5-6 dentículos marginais e sem dentículos submarginais; (3) tergos III-VII com tubérculo mediano; (4) margens laterais dos segmentos abdominais III-IX expandidos; (5) brânquias operculares ovais, sem a linha pouco esclerotizada, com uma longa lamela ventral; (6) espinhos na margem posterior dos tergos nos segmentos I-IV e VII-X; (7) fórmula brânquial 5/4/4/2/1.

Discussão:Apesar da similaridade entre as ninfas de $T$. rondoniensise algumas espécies de Tricorythodes, como a ausência de uma linha fracamente esclerotizada na brânquia opercular, é possível observar que essa espécie apresenta as características típicas de Tricorythopsis, tais como a presença de pequenos espinhos na margem posterior dos segmentos I-IV e VII-X e fórmula branquial 5/4/4/2/1. Dessa forma, essas características justificam a nova combinação proposta no presente artigo (e.g., Molineri 2001). Corroborando essa nova combinação, uma espécie recentemente descrita, Tricorythopsis spongicola Lima, Salles \& Pinheiro, 2011 também apresenta a brânquia opercular desprovida da conspícua linha fracamente esclerotizada (Lima et al. 2011).

Material Examinado: PF3: (1N) 13.xi.2009 e BR1: (1N) 09.x.2009.

\section{Tricorythopsis yucupe Dias, Salles \& Ferreira, 2008}

Estágios descritos: ninfa, subimago macho e imago fêmea. Biologia: As ninfas podem ser encontradas em raízes e pedras nos igarapés de laje com correnteza.

Distribuição: Brasil: Amazonas (Presidente Figueiredo), Roraima (Amajarí) (Dias et al. 2008); Venezuela: Bolivar (Tarota) (Molineri et al. 2011).

Material Examinado: PF8: (2N) 09.xi.2009. 


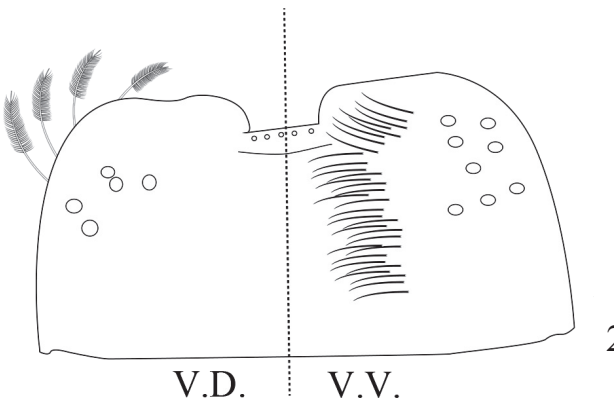

2
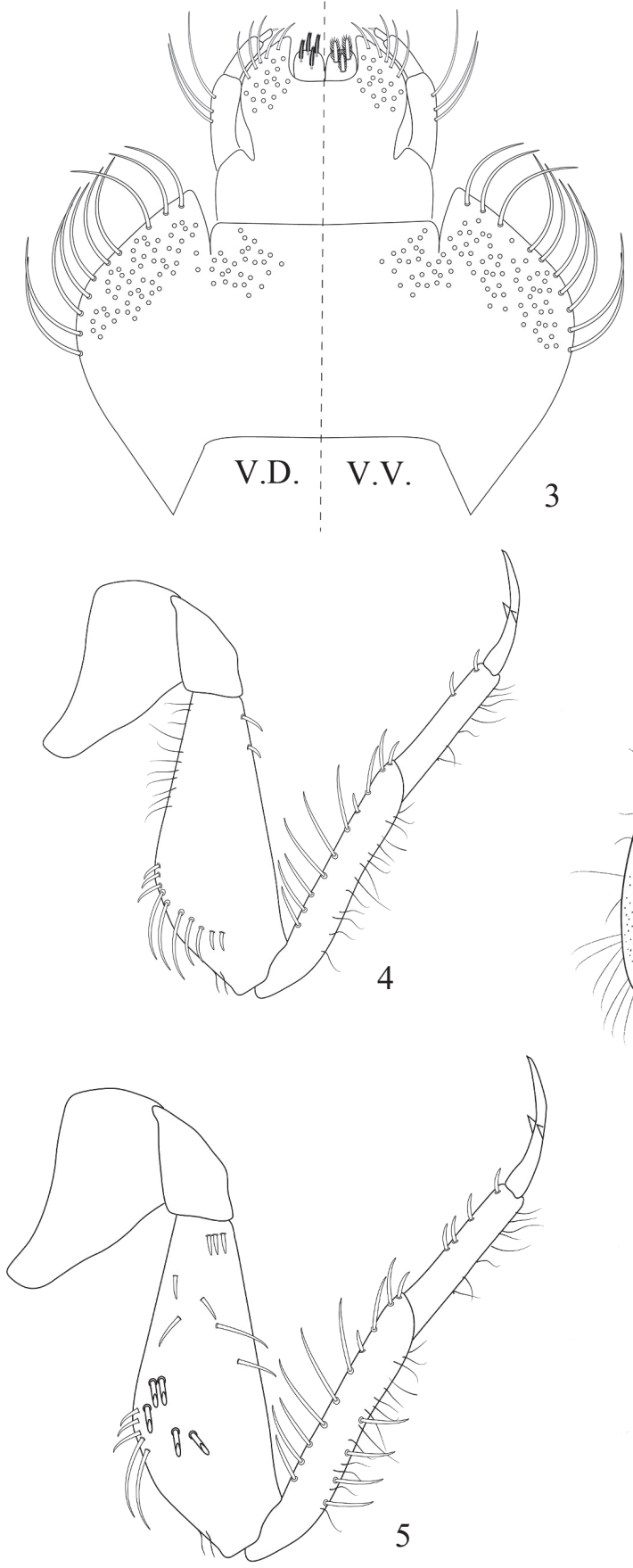

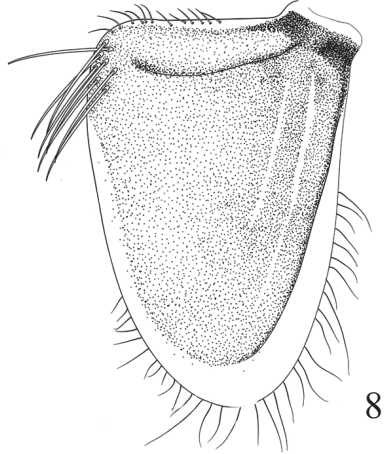

8
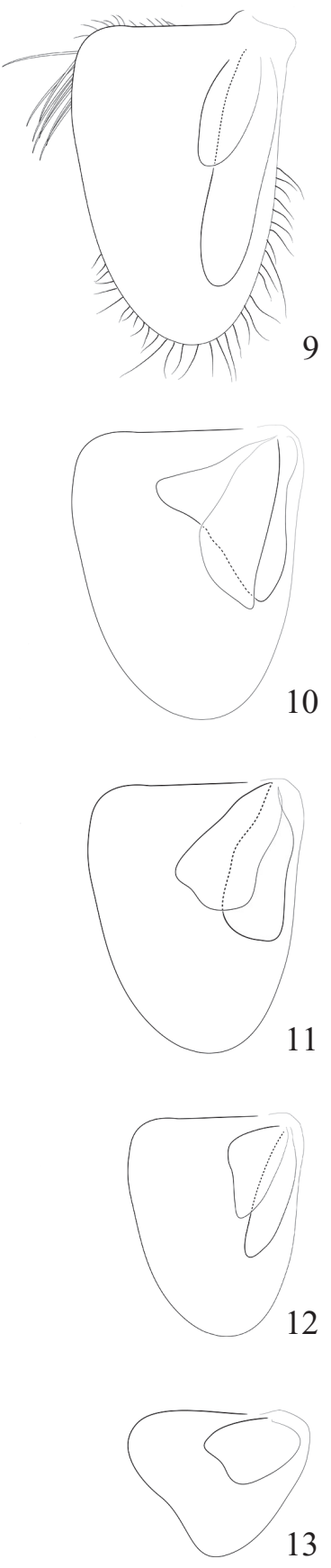

Figs. 2-13. Tricorythodes yapekuna sp nov.: (2) labro (vista dorsal e vista ventral); (3) lábio (v.d. e v.v.); (4) perna anterior; (5) perna mediana; (6) maxila; (7) abdome da ninfa (v.d.); (8) brânquia opercular (v.d.); (9) brânquia opercular (v.v.); (10-13) brânquias III-VI. 

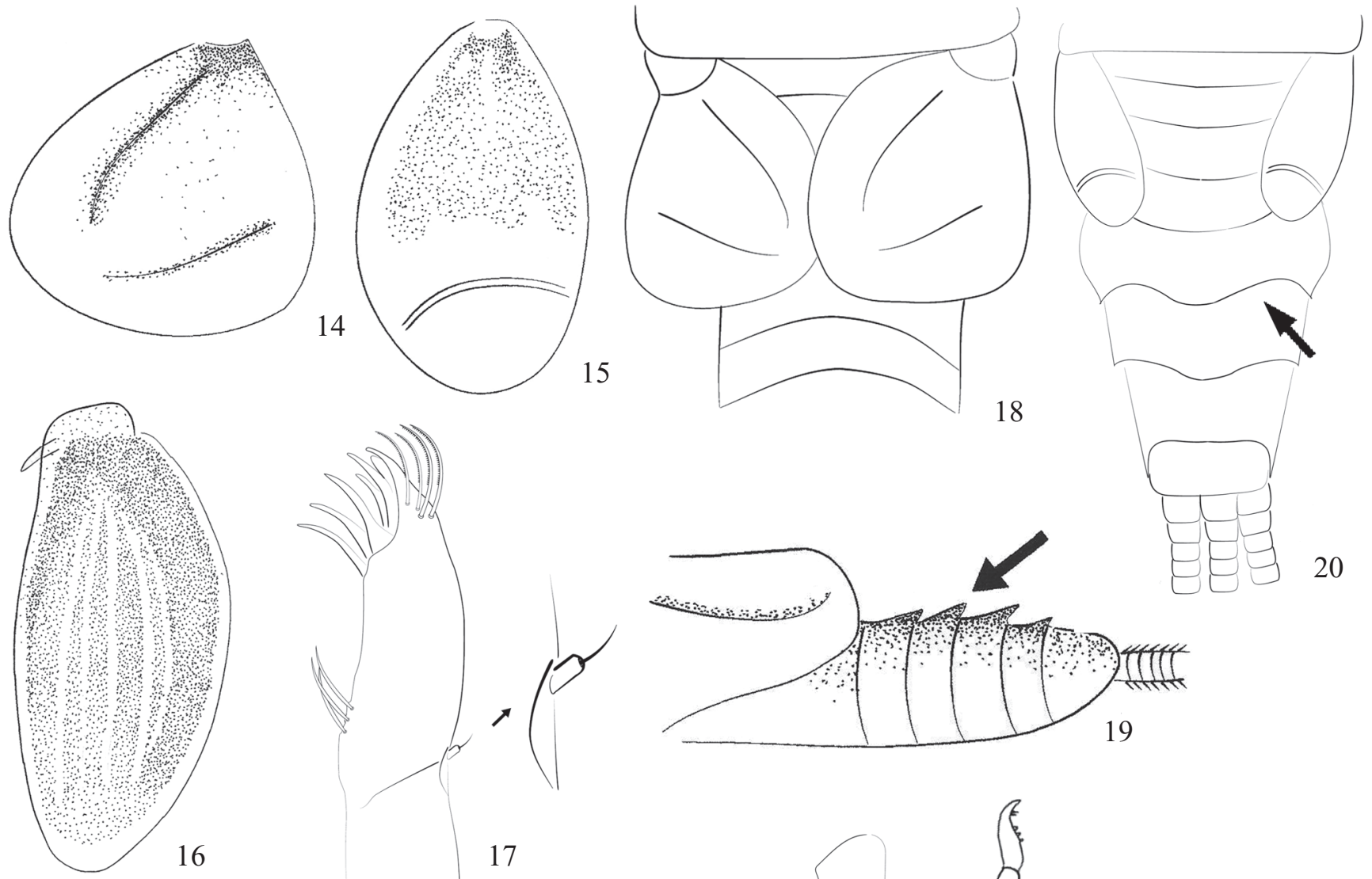

16

17
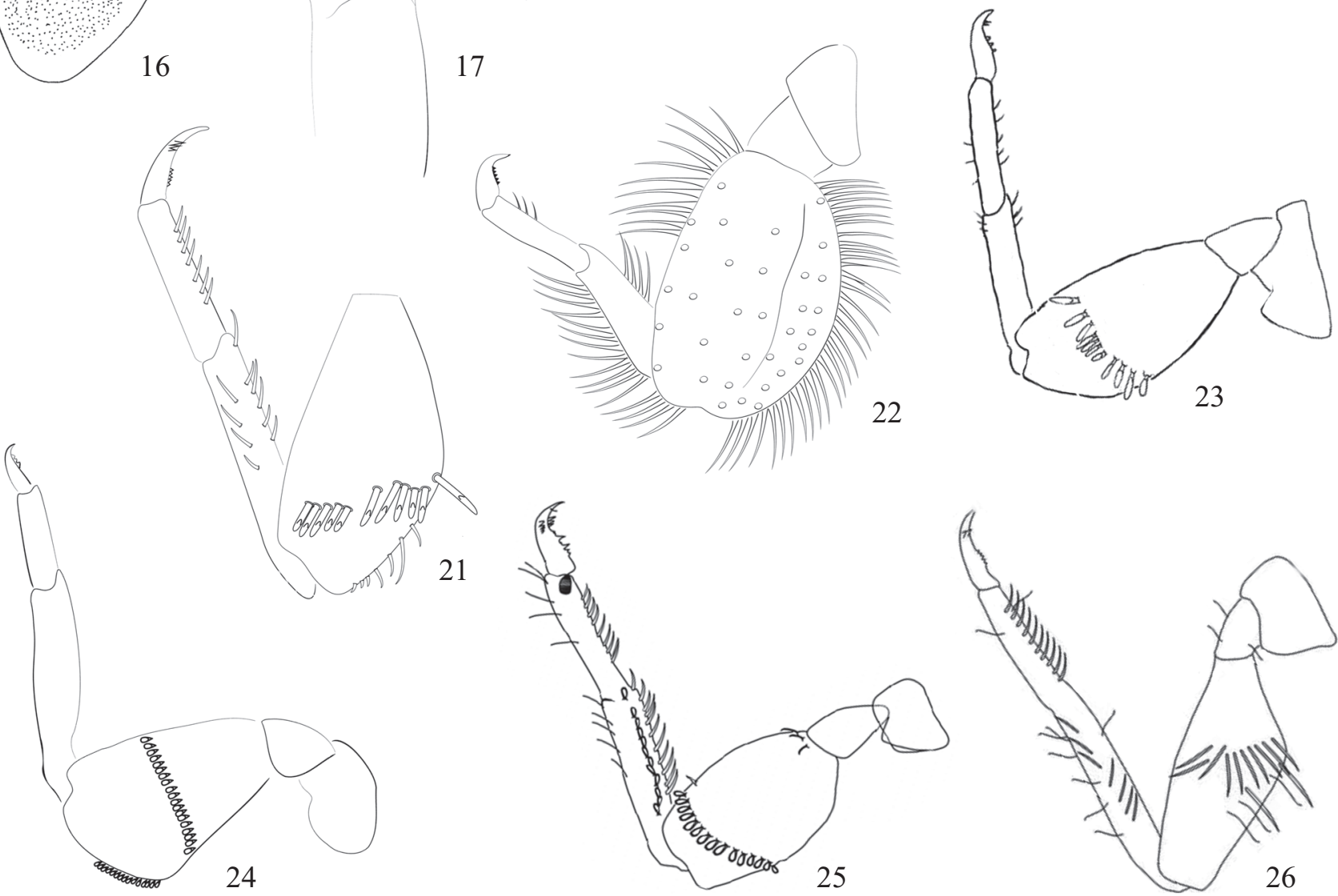

Figs. 14-26. 14-17. (14) brânquia opercular de Amanahyphes saguassu; (15) brânquia opercular de Tricorythopsis acara; (16) brânquia opercular de Leptohyphes populus; (17) maxila de Tricorythodes faeculopsis. 18-20. (18) abdome (v.d.) de Amanahyphes saguassu; (19) abdome (v.1.) de Tricorythopsis yucupe; (20) abdome (v.d.) de Tricorythopsis intercalatus. 21-26. (21) perna anterior de Tricorythopsis acara; (22) perna anterior de Tricorythopsis rondoniensis; (23) perna anterior de Tricorythopsis yucupe; (24) perna anterior de Leptohyphes populus; (25) perna anterior de Tricorythopsis intercalatus; (26) perna anterior de Tricorythodes faeculopsis. 
Chave para identificação dos gêneros e espécies de Leptohyphidae do Amazonas com base em ninfas

1. Brânquia opercular tão longa quanto larga (Fig. 14), com margens internas tocando-se ou muito próximas à região mediana do corpo (Fig. 18); brânquias presentes nos segmentos abdominais II-V .......... Amanahyphes saguassu

1'. Brânquia opercular triangular (Fig. 8) ou oval mais longa que larga (Fig. 15), margem interna não tocando a linha mediana (Fig. 19); brânquias presentes nos segmentos abdominais II-VI 2

2. Comprimento do corpo menor que $4 \mathrm{~mm}$; brânquia opercular frequentemente com uma linha transversal fracamente esclerotizada (Fig. 15); fórmula branquial 5/4/4/2/1 .....

Tricorythopsis .... 3

2'. Comprimento do corpo maior que $4 \mathrm{~mm}$, brânquia opercular sem linha transversal (Fig. 16); fórmula branquial não como acima

3. Fêmur estreito, pelo menos duas vezes mais longo que largo bordeado por cerdas de comprimento mediano (Fig. 21); garra tarsal com 5 dentículos marginais e 3+2 dentículos submarginais T. acara

3'. Fêmur largo ou circular, tão largo quanto longo, bordeado por cerdas de comprimento longo ou curto (Fig. 22); garra tarsal não como acima

... 4

4. Presença de tubérculos nos segmentos abdominais (Fig. 20); margem lateral dos segmentos abdominais expandidos de III-IX

5

4'. Ausência de tubérculos nos segmentos abdominais; margem lateral dos segmentos abdominais expandidos a partir do II

5. Tubérculos abdominais nos segmentos VII-IX; coxas anterior e mediana com projeção (Fig. 23); garra tarsal com 3-5 dentículos marginais e 1-2+1 dentículos submarginais T. yucupe

5 'Tubérculos abdominais nos segmentos III-VII; coxas sem projeção; garra tarsal com 5-6 dentículos marginais e sem dentículos submarginais T. rondoniensis

6. Garra tarsal com 9 dentículos marginais intercalados por dentículos largos e 4+3 dentículos submarginais (Fig. 25); presença de ondulações nas margens posteriores dos segmentos VI e VII (Fig. 19) .... T. intercalatus

6'. Garra tarsal com 3-5 dentículos marginais pequenos e 5 + 3-4 dentículos submarginais; ausência de ondulações nas margens posteriores dos segmentos abdominais ......

T. bahiensis

7. Brânquia opercular ovalada com projeção na base da lamela ventral (Fig. 16); fêmur margeado por cerdas curtas (Fig. 24) Leptohyphes (L. populus)

7'. Brânquia opercular triangular (Fig. 8); fêmur margeado por cerdas longas (Figs. 4-5) ......... Tricorythodes ...... 8

8. Palpo maxilar uniarticulado (Fig. 18); garras tarsais com 4-6 dentículos marginais e com cerda apical (Fig. 26) .. T. faeculopsis
8'. Palpo maxilar biarticulado (Fig. 6); garras tarsais sem dentículos marginais e um par de dentículos submarginais (Fig. 3) T. yapekuna sp. nov.

\section{AGRADECIMENTOS}

Ao Conselho Nacional de Desenvolvimento Científico e Tecnológico (CNPq) pelas bolsas e auxílios financeiros concedidos aos autores. Ao PRONEX-CNPq-FAPEAM e PPI INPA/MCT pelo apoio ao projeto de pesquisa. Ao Dr. Carlos Molineri pelas valiosas sugestões.

\section{REFERÊNCIAS}

Allen, R. K. 1973. New species of Leptohyphes Eaton (Ephemeroptera: Tricorythidae). Pan-Pacific Entomologist49: 363-372.

Allen, R. K. \& S. S. Roback. 1969. New species and records of New World Leptohyphinae (Ephemeroptera: Tricorythidae). Journal of the Kansas Entomological Society 42: 372-379.

Belmont, E. L. L; F. F. Salles \& N. Hamada, 2011. Three new species of Leptohyphidae (Insecta: Ephemeroptera) from Central Amazon, Brazil. Zootaxa 3047: 43-53.

Cummins, K.W.; R.W. Merritt\&P.C.N. Andrade.2005. The use of invertebrate functional groups to characterize ecosystem attributes in selected streams and rivers in south Brazil. Studies on Neotropical Fauna andEnvironment 40: 69-89.

Dias, L. G.; C. Molineri \& P. F. S. Ferreira. 2007. Ephemerelloidea (Insecta: Ephemeroptera) do Brasil. Papéis Avulsos de Zoologia 47: 213-244.

Dias, L. G.; F. F. Salles \& P. S. F. Ferreira.2008. New species of Tricorythopsis Traver (Ephemeroptera: Leptohyphidae) fromnorthernBrazil. Studieson Neotropical Fauna andEnvironment 43: 237-241.

Dias, L. G.; P. V. Cruz \& P. S. F. Ferreira. 2009. A new species of Tricorythodes Ulmer (Ephemeroptera: Leptohyphidae) from Northern Brazil. Annales de Limnologie 45: 127-129.

Domínguez, E.; C.Molineri; M. L. Pescador; M. D. Hubbard \& C. Nieto. 2006. Ephemeroptera of South America, Aquatic Biodiversity of Latin América. In: J. Adis, J. R Arias, G. Rueda-Delgado \& K. M. Wantzen (Eds.) Aquatic Biodiversity of Latin América. Volume 2, Sofia-Moscow, Pensoft, 642 p.

Edmunds Jr, G. F.; Jr; S. L. Jensen \& L. Berner. 1976. The mayflies of North and Central America. Minneapolis, University of Minnesota Press, $330 \mathrm{p}$.

Gonçalves, I. C. \& E. R. Da-Silva. 2010. Ephemeroptera, Leptohyphidae, Amanahyphes saguassu Salles e Molineri, 2006: First record from State of Pará, Brazil. Checklist 6: 170-171.

Hubbard, M. D. 1982. Catálogo abreviado de Ephemeroptera da América do Sul. Papéis Avulsos de Zoologia 34: 257-282.

Lima, L. R. C.; F. F. Salles \& U. S. Pinheiro. 2011. New species of Leptohyphidae (Ephemeroptera) from northeastern Brazil. Zootaxa 3050: 63 69.

Molineri, C. 2001. El gênero Tricorythopsis (Ephemeroptera: Leptohyphidae): nuevascombinaciones y descripción de nuevasespecies y estadios. Revista de La Sociedad Entomológica Argentina 60: 217-238.

Molineri, C. 2003. Revision of the South American species of Leptohyphes Eaton (Ephemeroptera: Leptohyphidae) with a key to thenymphs. Studies on Neotropical Fauna and Environment 38: 47-70.

Molineri, C. 2006. Phylogeny of the mayfly family Leptohyphidae (Insecta: Ephemeroptera) in South America. Systematic Entomology 31: 711-728.

Molineri, C. 2010. Las especies de Leptohyphidae (Ephemeroptera) de las yungas de Argentina y Bolivia: diagnosis, distribución y claves. Revista de La Sociedad Entomológica Argentina 69: 233-252.

Molineri, C.; M. E. Grillet; C. Nieto; E. Domínguez \& E. Guerrero. 2011. New species and records for the mayfly families Caenidae, Leptohyphidae and Coryphoridae (Ephemeroptera, Pannota) from 
Venezuelan Guayana's uplands. Zootaxa 2750: 39-50.

PPBio. 2011. Programa de Pesquisa em Biodiversidade - PPBio. Disponí-

vel em: http://ppbio.inpa.gov.br/Port/inventarios/ducke/(acessado em 02 de fevereiro de 2011).

Salles, F. F. \& C. Molineri. 2006. Amanahyphes saguassu, a new genus and species of Leptohyphidae (Ephemeroptera: Ephemerelloidea) from northern Brazil. Aquatic Insects 28: 1-12.

Salles, F. F.; R. Boldrini; J. C.Nascimento; E. Raimundi\& Y. Shimano. 2011. Ephemeroptera do Brasil. Disponível em: https://sites.google.com/site/ listaephemeropteradobrasil/(acessado em 20 de julho de 2011).

Received 8/11/2011; accepted 9/7/2012

Editor: Daniela Maeda Takiya 